\title{
3-D Spectroscopy with a Fourier Transform Spectrometer
}

\author{
J.P. Maillard \\ Institut d'Astrophysique de Paris - CNRS, Paris, France
}

\begin{abstract}
.
With the advent of bidimensional array detectors the throughput advantage of a Fourier Transform Spectrometer (FTS) can be used to create a new type of 3-D spectrometer. The classical multiplex property in the spectral domain of a FTS is multiplied by the number of pixel of the array. The points of the entrance field are all observed in parallel. After discussing the properties of this instrument, the coupling of the FTS of the CFH Telescope to a camera equipped with a NICMOS 3 array is described. With this combination, spectro-imaging in any bandpass between 1 and $2.5 \mu \mathrm{m}$ is possible within a circular 24" field of view, with a scale of 0.33 "/pixel, at seeing-limited spatial resolution. Any spectral resolution is choosable up to 30,000 . Illustrations are given by a study of the dark side of Venus at $1.27 \mu \mathrm{m}$ and of planetary nebulae at $2 \mu \mathrm{m}$. Many other objects can benefit from this observing mode in the near infrared. Further developments of this 3-D technique are discussed.
\end{abstract}

\section{Introduction}

Fourier Transform Spectrometers (FTS) are generally used for high resolution spectroscopy in the infrared. A large spectral range can be covered with a single detector, which expresses the multiplex property of this class of spectrometer. An enormous quantity of high resolution spectroscopic data, essentially in the infrared, on atomic and molecular species in laboratory and on various astronomical objects as well (Sun, planets, stars ...) have been obtained by FTSs in the last thirty years. Most of these spectra would have been impossible to record in the same amount of time with the same resolution by using conventional spectrometers. These results illustrate also an advantage in luminosity of the FTS. By integrating the flux from a laboratory or a stellar source over the circular aperture permitted by the Michelson interferometer which makes a FTS, very high spectral resolutions $\left(>10^{5}\right.$ ) are reachable without loss of light. That expresses the classical throughput advantage of an interferometric device. For example, the full étendue generated by a star behind a large telescope, spread by atmospheric turbulence, can be totally accepted by a FTS. This advantage is usable also to integrate over several arcseconds on extended objects like planets, planetary nebulae, galactic nuclei. Sometimes useful for objects with a low surface brightness, the contribution of different emitting parts in this case may be mixed in the resulting spectrum, which cannot be interpreted correctly. To avoid this confusion, independent spectra should be recorded, which multiplies the observing time by the number of points taken on the source. This ap- 
proach is rapidly too much time-consuming for complex and extended objects. A spectro-imager is the most appropriate solution. However, a long slit spectrograph, which gives only one space dimension, is not a satisfying response. For the study of a specific line, like the $\mathrm{H} \alpha$ emission in a galaxy, the most adapted solution, as demonstrated for a long time, is provided by an imaging Fabry-Pérot (Courtès 1960). On the other hand, for objects with a rich spectrum the FabryPérot is not well adapted. The free spectral range is too narrow. The ideal solution is an imaging spectrometer which can give simultaneously, on a large enough spectral range, the spectrum of all the resolved points of the source. In the visible, the TIGER spectrograph (Bacon 1988) has this ambition. In the near-infrared, with the advent of 2-D array detectors for the $1-2.5 \mu \mathrm{m}$ range, the same concept could be developed. That is not yet the case.

A different solution is offered by an imaging FTS, creating also a true 3-D spectrometer. The same method could work either in the visible with a CCD camera or beyond with an infrared array detector. However, FTSs have been developed in the infrared where they have been until today the only solution for highresolution spectroscopy on a large spectral range. Therefore, combined with thermal background considerations, an imaging FTS is an original technique to be put in operation for the $1-2.5 \mu \mathrm{m}$ region, where no equivalent solution exists. After studying the imaging and spectral properties of this new type of instrument, the first realization of this concept (Maillard \& Simons 1992) made by the coupling of a NICMOS-type camera to the FTS at the CFH Telescope facility is presented and illustrated. In conclusion, the future possibilities of this 3-D technique are discussed.

\section{Principle of an imaging FTS}

\subsection{Radial symmetry}

A Michelson interferometer, like a Fabry-Pérot, has a radial symmetry. The path difference is the same for all the points of the source with the same angle of incidence from the axis of the interferometer. Hence, a circular field is accessible.

\subsection{Stigmatism}

On the source side, an entrance collimator illuminates the interferometer with parallel beams. The interfering beams are collected by the output collimator, creating a stigmatic relationship between the object and the image planes. By placing a detector array in the output focal plane of a FTS adapted to a telescope, the entrance circular field is imaged on the array and each pixel works as a single detector matched to the corresponding point on the sky.

\subsection{Step-by-step scanning}

Retrieving the spectral information from images supposes to record the interferogram generated by the source point imaged on each pixel. The path difference has to be scanned. However, a constraint is imposed as imaging array detectors are integrating devices. Hence, 3-D spectroscopy with a FTS is only compatible with a step-by-step scannning of the path difference, a well-known technique in Fourier spectroscopy. The path difference changes by equal steps. Recording 


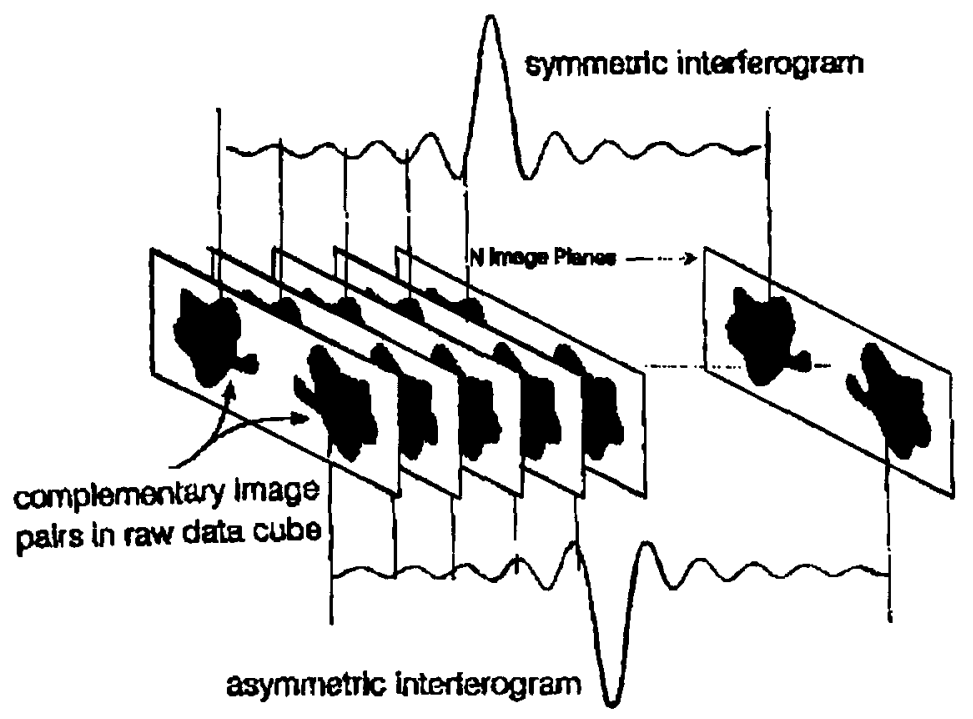

Figure 1. Cube structure of raw imaging FTS data, from which complementary image pairs of a same entrance point, simulated by a circle, create a symmetric $(S)$ and an asymmetric $(A)$ interferogram.

data in this mode means, after each mirror motion, while the path difference is stationary, integrating on the array and then recording a frame which at the end generates a data cube of interferograms (Fig. 1). Fast scanning FTSs, which are based on a continuous motion of the moving mirror, are not adapted to an imaging mode.

\subsection{Inverse transformation}

The signal of the same pixel on each frame forms an independent interferogram. These interferograms, sampled by the step-by-step scanning, have to be transformed individually. They give a sampled spectrum for each pixel. Then, a spectral data cube is constituted with the same spatial elements. Final product of a true 3-D spectrometer, each plan of this cube is a monochromatic image of the total field. The transformation does not introduce any geometric error.

\section{Characteristics of an imaging FTS}

The characteristics which define a spectro-imaging device are the spatial and spectral resolution combined to the spatial and spectral coverage obtainable in a single observation.

\subsection{Spectral resolution}

Increasing the path difference increases the spectral resolution. In imaging mode the requirement is that, for an off-axis beam, the angular dispersion be less than the desired spectral resolution. The maximum spectral resolution is a function 
of the field of view of the FTS. Let an interferometer be illuminated with a monochromatic source of wavenumber $\sigma_{0}$. If $\delta_{0}$ is the nominal path difference, for a beam with an angle of incidence of $\theta$ in the interferometer, the path difference is $\delta=\delta_{0} \cos \theta$. The apparent wavenumber of this beam is:

$$
\sigma_{\theta}=\frac{\sigma_{0}}{\cos \theta} \simeq \sigma_{0}\left(1+\frac{\theta^{2}}{2}\right)
$$

from which

$$
\frac{\left(\sigma_{\theta}-\sigma_{0}\right)}{\sigma_{0}}=\frac{\theta^{2}}{2}
$$

With $\mathrm{d} \sigma$ being the desired limit of resolution and $R$ the corresponding resolving power, the spectro-imaging condition is:

$$
\frac{\left(\sigma_{\theta_{\text {max }}}-\sigma_{0}\right)}{\sigma_{0}}=\frac{\mathrm{d} \sigma}{\sigma_{0}} \Rightarrow R=\frac{2}{\theta_{\max }^{2}}
$$

$¿$ From the radial symmetry of the Michelson interferometer, this last equality can be transformed to show $\phi$, the total angular field on the sky when the FTS is matched to a telescope. The relation becomes:

$$
R=\frac{8}{\phi^{2}} \frac{1}{m^{2}}=\frac{8}{\phi^{2}}\left(\frac{d}{D}\right)^{2}
$$

with $m$ the angular magnification, $D$ the telescope diameter and $d$ the diameter of the beams in the FTS. This relation imposes the maximum spectral resolution from the field optically accepted by the FTS behind a telescope. Inversely, in the design of an imaging FTS for a specific telescope, this relation determines the diameter of the beamsplitters in the FTS needed to reach a given spectral resolution over the desirable field of view. In a project of FTS for a 8-m telescope (Maillard 1988) is indicated that a field of 1.5 arcmin in diameter could be studied in one scan with a resolving power of 1000 , providing beams of only 50 $\mathrm{mm}$ in the FTS. A spectral resolution of $10^{4}$ is reached by limiting the field of view to $\simeq 30$ arcsec.

Within these limits, an important feature which is unique to a FTS, is kept intact by the possibility of varying continously the spectral resolution to adapt it to the scientific problem, just by changing the number of frames to record.

\subsection{Field of view}

The size of the array and the choice of the spatial sampling define the potential field of view. Current $\mathrm{HgCdTe}$ arrays for the $1-2.5 \mu \mathrm{m}$ range are of $256 \times 256$ pixels. With a plate scale of 0.25 arcsec/pixel the maximum field of view offers by the array is $\sim 1$ arcmin, which is adapted to the field size indicated in the previous paragraph behind a $8-\mathrm{m}$ telescope. Behind a $4-\mathrm{m}$ the same FTS could accept 2 arcmins and a resolution of $10^{4}$. In this case, the plate scale should be of $0.5 \mathrm{arcsec} / \mathrm{pixel}$, which remains acceptable. However, the optical elements of the interferometer have to be adapted to accept this field. Modern FTSs are currently made around an interferometer equipped with cat's eye retroreflectors, an optical system which has the advantage for large path differences of maintaining the alignment during scanning. In this case, the maximum field is limited 
by the secondary mirror in each cat's eye as the entrance field is focused on it. The focal length of the primary mirrors has to be adapted to comply with the required field.

In a FTS with a retroreflector in each arm, two complementary images of the entrance field are formed. In a project of imaging FTS for the HST (Hall 1986), two cameras, one for each output, were supposed to be used. Hence, the potential field is directly defined by the array size. In the instrument which is described below, for the sake of simplicity, a single camera is used. Therefore, an interface is needed to put, side-by-side, the two images onto the array. In this case, the usable field is a quarter of the field offers by the camera.

\subsection{Spatial resolution}

Providing that the optical aberrations of an off-axis source point are smaller than the spatial sampling, which corresponds to the pixel size of the array, a sharpening of the images is possible. That supposes in the data processing a recentering of a frame with respect to the next, to correct for the guiding errors, the flexures and the turbulent motions of the images, before extracting the interferograms from which the spectra are retrieved. The ultimate spatial resolution depends on the quality of this operation.

\subsection{Spectral coverage}

As in a standard FTS, the spectral coverage in one observation is determined by the bandpass of the cold filter put in the camera. The multiplex properties of the FTS are preserved. Even, the same spectral coverage is obtained for all the points of the field, which is not the case with TIGER for example, where the spectra of the points at the edge of the field are truncated. However, constraints are introduced by the total observing time which can be devoted to a scan, the required resolution and signal-to-noise considerations. Larger is the spectral range $\Delta \sigma$ smaller is the step size to sample the path difference needed to reach the limit of resolution $\mathrm{d} \sigma$. The resulting number of steps $N$ is also equal to the number of spectral elements in the spectrum.

$$
N=\frac{\Delta \sigma}{\mathrm{d} \sigma}
$$

The total observing time is

$$
T=N\left(\tau+t_{d}\right)
$$

with $\tau$ the integration time of each frame and $t_{d}$ the overhead time associated with its recording. $t_{d}$ should be as short as possible but cannot be negligible. $\tau$ is determined to get photon noise-limited conditions on the images of the source through the chosen filter, without saturation. From a maximum scanning time ( 3 hours seems a practical limit) $N$ is imposed and therefore $\mathrm{d} \sigma$. A higher resolution implies to limit the spectral range, i.e. a narrower filter.

\subsection{Flexibility}

In conclusion, an imaging FTS has the most flexibility compared to any other spectro-imaging devices, like long slit grating spectrometer, scanning FabryPérot, multi-fiber spectrograph and TIGER-type spectrograph. The spectral 
resolution is choosable, up to the maximum value imposed by the size of the field. The field of view can be conveniently of half an arcmin and the spectral resolution up to several times 10,000 , even behind a 8 -m class telescope. The spectral range can be chosen in principle as large as necessary, independently of the size of the array as is the case for grating spectrographs. The spatial resolution can be close of seeing-limited conditions on short exposure times.

Such unique advantages led us to realize the coupling of the NICMOS camera (Redeye) recently developed at the Canada-France-Hawaii Telescope (Clark et al. 1994) to the FTS which is part of the telescope instrumentation (Maillard \& Michel 1982), and is currently used for high-resolution spectroscopy with InSb mono-detectors in the $1-5.4 \mu \mathrm{m}$ range. This instrument is a step-by-step scanning interferometer, and so it was directly adaptable to an imaging mode. Such combination is the first astronomical 3-D spectrometer of this type.

\section{Design of the imaging CFHT-FTS}

\subsection{Field and resolution}

The CFHT-FTS is adapted, for maximum optical efficiency, to the $\mathrm{f} / 35$ infrared Cassegrain focus of the $3.6 \mathrm{~m}$ telescope. The interferometer uses cat's eye retroreflectors in each arm to provide the symmetric and asymmetric outputs. The field of view is equal to 24 arcsecs, determined by the size of the secondary mirror in the cat's eyes. In practice, a full field of view of 20 arcsecs is usable. The maximum limit of resolution allowed by the interferometer by scanning the total path difference is $5 \times 10^{5}$ at $2 \mu \mathrm{m}$. With the above field, from Equ. 4 the maximum spectral resolution is $\simeq 30,000$.

\subsection{Camera interfacing}

The development of the imaging mode has been conceived in such a way that the FTS can be easily returned to its standard mode of operation. An optical interface box is bolted to the FTS's existing detector mounting plate instead of the InSb dewars. The basic function of this interface box is to provide a means of bringing the two images of the telescope's focal plane produced by the FTS, onto a single array. The final scale on the detector is $0.33 \mathrm{arcsec} / \mathrm{pixel}$. The field corresponds to a circular area of 73 pixels in diameter. The symmetric and the asymmetric images are each placed on one quadrant of the array. Specifically for this application, a cold mask with two apertures of the size of the field is placed in the focal plane of Redeye to further reduce the off-axis background emission coming from the interface. After a careful focusing of the interface, images of a star through the whole assembly with a FWHM of 2 pixels are obtained $(0.66$ arcsec).

\subsection{Data acquisition}

The size of a frame is determined by the reading of two quadrants of the detector array - limited to $100 \times 256$ pixels with 16 bit words per line. Determining the zero path difference (hereafter ZPD) is the first operation before an interferogram can be recorded. After locking onto $\mathrm{ZPD}$, the target is acquired in the field of 
view. The FTS mirror carriage is then backed away from ZPD and a forward scan sequence is initiated.

The path difference is controlled in position from the error signal produced by a monomode stabilized He-Ne laser, and can be changed by steps that are multiple of $1 / 8$ of its wavelength. The optimum step size depends on the bandpass of the filter put in front of the camera. The integration time is generally chosen to yield photon-noise limited images. Hence, it depends directly on the brightness of the source through the chosen filter. The mirror motion is very fast $(<5 \mathrm{~ms})$ compared to the total overhead time made by the readout of the array, the storage of a frame plus the dialog through the computer between the camera and the FTS. The dead time currently obtained is $2.5 \mathrm{~s}$. This value is very critical and imposes a limitation on the total number of frames which can be recorded, which has a consequence on the final limit of resolution. The number of frames in a raw data cube has typically been around 500, and pushed to 1000. In this case, over a $250 \mathrm{~cm}^{-1}$ bandpass (which is for example $1 / 4$ of the full $K$ band) the resolution is limited to $0.25 \mathrm{~cm}^{-1}$ (i.e. 20,000 or $15 \mathrm{~km} / \mathrm{s}$ at $2 \mu \mathrm{m}$ ). The dead time becomes already of $\simeq 40 \mathrm{~min}$. For a total observing time of three hours, the integration time per frame is at most equal to $\sim 8.3 \mathrm{~s}$. These considerations determine the ultimate performances of this mode. There is no simple answer as they depend on the nature of the spectrum (emission or absorption), the filter bandpass, the spectral resolution and the final spatial resolution.

\subsection{Data processing}

After straightforward digital image processing (flat fielding and bias subtraction) of each frame of the raw data cube, the successive operations in detail are: i) splitting of the frames in two half-frames (one called $S$ for symmetrical, the other $\boldsymbol{A}$ for asymmetrical), and flipping of one half about the y-axis, ii) recentering each field with respect to the first image in the raw data cube, iii) cosmic ray removal, iv) creation of the $S-A$ difference which will be the final interferogram or alternatively the normalized ratio $(S-A) /(S+A)$ to correct for transparency variations, $v$ ) inversion of each interferogram through a FFT routine by producing power spectra at each pixel position, vi) creation of a data cube $(x, y, \sigma)$ from which monochromatic images can be extracted, and also individual spectra by summation over several pixels. From the spatial resolution given above, the use of a 0.66 arcsecond software aperture is possible. In conclusion, within the 20 arcsec circular field, $\simeq 720$ independent spectra can be recorded simultaneously.

\section{Results}

\subsection{Dark side of Venus}

Venus' atmosphere presents several narrow windows between 1 and $2.2 \mu \mathrm{m}$, detectable on the night side only, which make it possible to probe deep into the atmosphere, below the cloud deck. High resolution spectroscopy of these windows at $1.18,1.27,1.7$ and $2.2 \mu \mathrm{m}$ was obtained with the FTS in its standard observing mode (Bézard et al. 1990), with a 5 arcsec aperture integrating the 


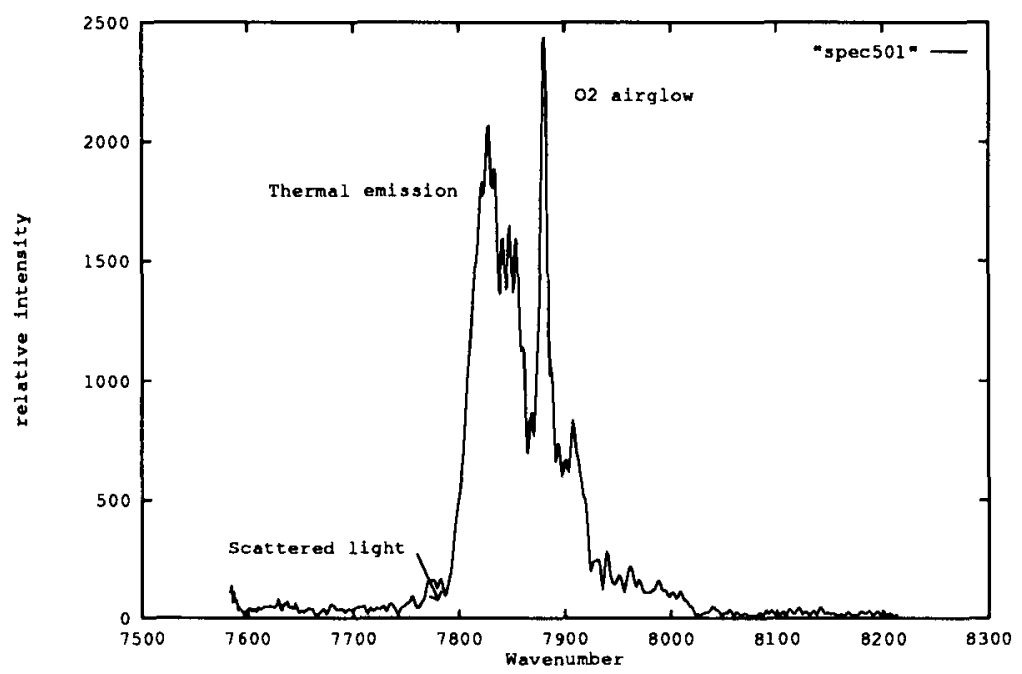

Figure 2. One raw spectrum of the dark side of Venus at $1.27 \mu \mathrm{m}$ $\left(7880 \mathrm{~cm}^{-1}\right)$ over 1 ", at the center of the 24 " field, taken on March 8 , 1993 (UT). The resolution is equal to 1800 . The field was tangent to the limb, centered on the northern hemisphere of the planetary disk. Three components are seen: the $\mathrm{O}_{2}$ airglow emission $\left({ }^{1} \Delta\right.$ band with $P, Q$ and $R$ branches), the thermal emission window from Venus low atmosphere, and the scattered light from the bright side.

flux on one position of the dark side, leading to interesting new results on Venus' atmosphere composition. At $1.27 \mu \mathrm{m}$ the main feature is an emission band of oxygen, of a completely different origin, taking place in the upper atmosphere, at $90 \mathrm{~km}$, resulting in a nightglow. The study of the morphology of this emission is a typical problem for a 3-D spectrometer.

This band was observed during two runs with the imaging FTS (Maillard et al. 1993). Fig. 2 shows the example of one spectrum at $1.27 \mu \mathrm{m}$ extracted from the data after pre-processing. The full processing supposes subtracting a map of the scattered light coming from Venus' bright crescent. This map is obtained by generating images in a portion of the filter bandpass outside Venus' emissions. By modelling the $\mathrm{O}_{2}$ emission $\left(\mathrm{T}_{\text {rot }}=190 \mathrm{~K}\right)$, the thermal emission can be separated from the airglow emission and calibrated maps of each contribution can be made. The thermal emission is essentially uniform over the field while the oxygen nightglow is strongly variable. For that day, the emission was present over all the field with a variation of more than a factor 2 . These data represent the ideal tool to study this highly variable phenomenon, related to the atmospheric circulation of Venus. More data over several consecutive days are needed to build a model of circulation. Comparable analysis can be carried out on water vapor from the $1.18 \mu \mathrm{m}$ window and at $2.45 \mu \mathrm{m}$ on the $\mathrm{SO}_{2}$ and OCS abundance distribution. 


\subsection{Other objects}

Data have been obtained also on Jupiter at $2.15 \mu \mathrm{m}$, with the field aperture centered respectively on both polar regions. Position of the cloud top at the poles, on the zones and the bands, vertical distribution of $\mathrm{CH}_{4}$, can be studied from these data at a spectral resolution of $\sim 1500$.

During a run in Nov. 1993, two planetary nebulae, NGC 7027 and CRL 2688 were observed. NGC 7027 is the prototype of a young planetary nebula with the remnants of its red giant circumstellar envelope, and an outer molecular shell surrounding the central, ionized region. Its structures extend over $\sim 20$ arcsecs, well-suited to our field of view. The integrated spectrum in the infrared (Smith et al. 1981) shows a rich variety of emission lines from neutral and ionized atoms, strong recombination lines of hydrogen, as well as molecular hydrogen. We therefore chose a filter covering simultaneously $\mathrm{B} \gamma\left(4616.6 \mathrm{~cm}^{-1}\right), 1-0$ $\mathrm{S}(1)$ of $\mathrm{H}_{2}\left(4712.9 \mathrm{~cm}^{-1}\right)$, HeI lines $\left(4733.4\right.$ and $\left.4857.5 \mathrm{~cm}^{-1}\right)$ and HeII line $\left(4568.1 \mathrm{~cm}^{-1}\right)$. The new data make possible to clearly separate the regions of excitation of each species. The same filter was used for CRL 2688 even if only $\mathrm{H}_{2}$ was present beside the continuum. Up to 6 lines of the $1-0$ and $2-1$ band were observable all together. After reduction, monochromatic images in the prominent emission lines were obtained. The velocity field of each species has been extracted, in particular for $\mathrm{H}_{2}$, which is a unique aspect of these data. A first analysis is presented by Cox et al. (1994) at this Conference.

\section{Conclusions}

\subsection{Scientific targets}

The four planets (Venus, Mars, Jupiter and Saturn) are well-adapted targets to study the spatial variations of their atmospheric composition. Several planetary nebulae like CRL 618, BD+30 3639 are other potential targets to study the kinematics and the excitation conditions of their molecular envelope. More generally, the 1 to $2.5 \mu \mathrm{m}$ range gives access to many $\mathrm{H}, \mathrm{H}_{2}, \mathrm{HeI}$ lines and also to FeII and $\mathrm{CO}$ to mention the most common spectroscopic indicators. With these lines many objects can benefit from this mode: reflection nebulae, open clusters, the Galactic Center, near-by starburst galaxies (e.g. M82, NGC253).

\subsection{Future developments}

With the instrument presented here, no further instrumental developments are possible. In particular, the usable field which determines the choice of the scientific targets cannot be changed. However, progress in the data acquisition are still possible by reducing the overhead time which would improve the overall efficiency of the instrument. With the increased capacity of the computing machines larger data cubes than the current 1000 frames can be recorded, allowing higher resolution or repeated observations to increase the signal-to-noise ratio, without leading to an impracticable processing time. For a larger field, a higher spatial resolution or the capability of working at longer wavelength, all characteristics which are compatible with this technique, a new instrument should be developed. 
Field of view As discussed in section 3.2 an imaging FTS can be easily designed to accept a field of view much larger than the 24 arcsecs of the CFHT-FTS, behind a 4-m class telescope. With the advent of $1024 \times 1024 \mathrm{HgCdTe}$ arrays a field of almost 3 arcmins can be covered (with the 0.33 arcsec/pixel and using a single camera). However, the main problem will be shortening the overhead time (to be kept $\leq 2.5 \mathrm{~s}$ ) for reading and storing frames more than 20 times bigger than the current frames.

Spatial resolution An imaging FTS could be an auxiliary instrument of an adaptive optics system, opening a completely new domain of spectro-imagery at high spatial resolution in the near infrared. However, the problem of the overhead time mentioned above would be the same on a smaller field.

Spectral coverage At last, the spectral coverage is limited in the current instrument to $2.5 \mu \mathrm{m}$ because the FTS is at room temperature. Behind a cryogenically cooled-FTS, which is technically feasible, an InSb array detector could be installed, extending the capabilities of spectro-imagery to $5.4 \mu \mathrm{m}$, which would realize all the capabilities of this new 3-D technique.

\section{Discussion}

G. Monnet: Would it be correct to say that your beautiful technique is in fact equivalent to synthetizing a Fabry-Pérot in real time, where the Michelson mirror step gives the Fabry-Pérot gap and the number of steps the finesse?

J. P. Maillard: Being in Marseille, everything has to be compared to the FabryPérot! The expression given in sect. 3.4 of this paper (number of steps $\mathrm{N}=$ $\Delta \sigma / \mathrm{d} \sigma)$ gives exactly the response to your question, knowing that the reflection finesse of a Fabry-Pérot is $\Delta \lambda / \mathrm{d} \lambda$, the ratio of the inter-order spacing to the width of the peak. From this comparison, the interest of the FTS can be expressed by the capability of reaching easily the equivalent of finesse of 1000 and possibly more.

P. Mein: Image motions or transparency fluctuations may occur during the scan. Can you correct such effects by using the 2-D interferograms?

J. P. Maillard: Image motions are corrected in the data processing (Sect. 3.3) by recentering each frame with respect to the next one. That can be done to the fraction of pixel. Regarding transparency fluctuations during a scan, they can be corrected from the simultaneous recording of the two outputs of the interferometer which is made (see Sect. 4.4).

N. Douglas: Following the question of correcting for errors in spatial position, time variations during a scan are possibly more problematic. I am wondering if the time-variation during the cometary collision on Jupiter would make this observation impossible.

J. P. Maillard: Any spectro-imaging work on Jupiter is confronted with the fast rotation of the planet. The duration of a scan has to be estimated in relation to the spatial resolution which is expected. It turns out that a scan cannot be much longer than 10 to 15 minutes, which supposes to limit the spectral resolution to 
make the minimum number of steps. For the specific observations you mention, they should be post-impact observations to look at the chemical modifications of the atmosphere. It can be assumed that on this span of time the conditions will be stable.

N. Douglas: Are you happy to use the power spectrum or are you considering taking the extra effort to correct the phase and thus improve the $S / N$ ?

J. P. Maillard: The use of the power spectrum has been useful to test and develop the method. It makes the data reduction faster. The plan is effectively to make this extra effort to correct the phase, and preliminary tests have been already done. The interferograms contain all the information needed as they are recorded before $Z P D$. The main gain is an improvement of the spectral resolution and a linear relation when coadding spectra.

$N$. Douglas: Comment: for clarity, your spectra neither use nor require registration of the zero path length since you use the power spectrum as instrumental profile.

J. P. Maillard: At any rate, the recording of ZPD is required to reconstruct the continuum of the spectra. Ideally, the interferograms should be recorded by starting exactly at ZPD. Like that an FT in amplitude could be used. With a step-by-step scanning of the path difference that is not easy to have a sampling just at ZPD. In addition, in imaging mode, ZPD is not exactly the same for all the points of the field because of the optical defaults of the wavefronts. I chose to record a minimum of $\mathbf{5 0}$ frames before ZPD instead of symmetrical interferograms. With this manner, the continuum is reconstructed correctly in a power spectrum; for the same number of samples the resolution is higher (at the expense, however, of secondary wiggles in the instrumental profile); later on, it will be possible to reprocess the interferograms to correct the phase.

Jockers: How does the imaging FTS compare to an infrared imager employing a Fabry-Pérot like, e.g. IRAC2 of ESO?

J. P. Maillard: The full answer is given in the paper. To summarize, the imaging Fabry-Pérot is to be preferred for the study of a single emission line (e.g. the $\mathrm{H}_{2}$ line at $2.12 \mu \mathrm{m}$ ) on a field of $\sim 1$ arcmin with a resolution of $\sim 10^{3}$. It makes a compact instrument, easy to adapt on an existing IR camera. The imaging FTS is a more complex instrument. In return, it offers all the flexibility for choosing the spectral range on a large domain, the spectral resolution, to have access simultaneously to several lines, in emission or in absorption.

\section{References}

Bacon, R., Adam, G., Baranne, A., Courtes, G., Dubet, D., Dubois, J. P., Georgelin, Y., Monnet, G., Pecontal, E., and Urios, J., 1988, in Very Large Telescope and their Instrumentation, ESO Conference No. 30, vol. II, p. 1185

Bézard, B., de Bergh, C., Crisp., D., and Maillard, J- P, 1990, Nature, 345, 508 Clark, C. C., Simons, D. A., and Massey, S., 1994, in Infrared Astronomy with Arrays: the next generation, Ian McLean (ed), Kluwer, vol. 190, 283 
Courtes, G., 1960, Ann. d'A strophysique, 23, 96

Cox, P., Maillard, J.P., Forveille, T., Simons, D., Guilloteau, S., Huggins, P.J., Omont, A., and Bachiller, R., 1994, this conference

Maillard, J. P., 1988, in Very Large Telescope and their Instrumentation, ESO Conference No. 30, vol. II, p. 1243

Maillard, J. P. and Michel, G., 1982, in Instrumentation for Astronomy with Large Optical Telescopes, IAU Colloquium No. 67, C. M. Humphries (ed), D. Reidel (Pub), vol. 92, 213

Maillard, J. P. and Simons, D., 1992, in Solar Physics and Astrophysics at Interferometric Resolution, ESA Workshop, L. Damé and T. D Guyenne (ed), ESA SP-344, 205

Maillard, J. P., Bézard, B., Domisse, L., Crisp, D. and Simons, D., 1993, B.A.A.S, 25, 1095

Smith, H. A., Larson, H. P., and Fink, U., 1981, Ap. J., 244, 835 